\title{
IMPACT OF ROOTING PROMOTER (PHOS ROOT-X) WITH OR WITHOUT USING IBA ON ROOTING OF TERMINAL AND MIDDLE CUTTINGS OF ROSEMARY (ROSMARINUS OFFICINALIS L.)
}

\author{
F.F. Lasheen ${ }^{*}$, M. Hewidy ${ }^{*}$ and O.N.K. Emam ${ }^{* *}$ \\ * Horticulture Department, Faculty of Agriculture, Ain Shams University, Egypt \\ ** Horticulture Department, Faculty of Agriculture, Beni-Suif University, Egypt
}

\begin{abstract}
This study was carried out in the Ornamental Plants Farm, Horticulture Department, Faculty of Agriculture, Ain Shams University, Cairo, Egypt. Two individual experiments were done. The first experiment aimed to study the effect of Phos root-x as newly rooting promoter at different rates $\left(0,0.5,1,1.5,2,2.5\right.$ and $\left.3 \mathrm{~cm}^{3} / 1\right)$ on rooting of the terminal cuttings of rosemary plants. This study was carried out on $20^{\text {th }}$ of July during the two seasons of 2019 and 2020. The experiment consisted of 7 treatments with 3 replicates, every replicate contained 3 cuttings per treatment. Data collected refer to that the $2.5 \mathrm{~cm}^{3} / 1$ of the newly rooting promotors were the best. Chemical analysis of terminal cutting refers to a decreasing in $\mathrm{C} / \mathrm{N}$ ratio of terminal cuttings with increasing of Phos root- $\mathrm{X}$ concentrations. The second experiment was done on $25^{\text {th }}$ of October in the same seasons, 2019 and 2020 for both terminal and middle cuttings and treated with Phos root-x complex with or without IBA, the treatments were $0,1,2,3 \mathrm{~cm}^{3} / 1$ of Phos root-x with or without IBA at $0,100,500 \mathrm{ppm}$ in combination. The experiment consisted of 24 treatments; each treatment consisted of three replicates with 3 cuttings per replicate. Data showed that for all parameters the best types for rooting was terminal cuttings, and a higher concentration of phos root$x$ favored better results especially with $3 \mathrm{~cm}^{3} / 1$ level. These results may be due to the higher concentrations of amino acids complex inside the newly rooting promotor compound (Phos root-x).
\end{abstract}

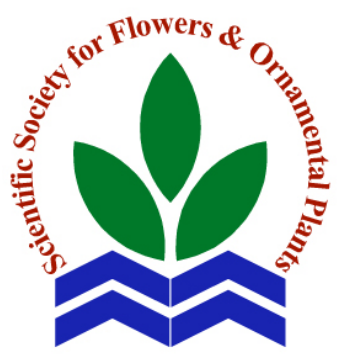

Scientific J. Flowers \& Ornamental Plants, 8(1):153-163 (2021).

Received:

$1 / 2 / 2021$

Accepted:

$6 / 3 / 2021$

Corresponding author: F.F. Lasheen fawzy_lasheen@agr.asu.edu.eg

Key words: rosemary, Rosmarinus officinalis L., amino acid, rooting, cuttings, IBA.

\section{INTRODUCTION}

Rosemary (Rosmarinus officinalis L.) belongs to Family Lamiaceae, it is an evergreen, perennial shrub that is native to Mediterranean region. Rosemary is used not only in perfumes and as food spice (Moss et al., 2003), but also in medicine (Miguel et al., 2007). The whole plant was reported to possess several medicinal properties like antiseptic, antispasmodic, carminative, antinervine spasmodic, astringent and tonic (Grieve, 1984; Polumin and Huxley, 1987; Chakaravati and Raghuvanshi, 2005). It was also used as a natural antimicrobial and antifungal against Escherichia coli, Pseudomonus, Aspergillus, Staphylococcus or as an insecticide (Duke, 2001). Besides, it was reported that the leaves have high antioxidant activity attributed to its phenolic compounds (Singh and Guleria, 2013), therefore, rosemary extracts are used as a preservative in the food industry (Bozin et al., 2007). Propagation by cutting is relatively cheap method because it overcomes the difficulties of multiplying by seeds for rosemary plants (Elhaak et al., 2015). Paradikovic et al. (2013) found that, 


\section{F.F. Lasheen et al.}

higher values of morphological properties such as plant height, number of leaves, fresh and dry weights of rosemary plants were recorded by using rooting powder "Rhizoopon 1" containing rooting powder auxin IBA at $0.5 \%$. Karimi et al. (2014) concluded that, the summit cuttings of Tymus satureioides improved rooting ability to $91 \%$ using IBA at $500 \mathrm{ppm}$. Elhaak (2015) reported that soaking rosemary cutting in low concentrations of IBA especially at 5 ppm level continuously found to be effective in cutting rooting and vigor.

Paulus et al. (2016) found that $2500 \mathrm{ppm}$ of IBA resulted in higher percentage of rooted cuttings (98\%) and favored the growth in height and accumulation of fresh and dry weights of above ground parts and roots, thus making more efficient rooting, enabling producer to obtain more developed seedlings in less time compared to the control treatment (0 ppm IBA) for rosemary plant. Massoud et al. (2017) concluded that using of natural products (minerals and amino acid compound) was better than IBA rooting auxin and resulted in enhancing rooting, vegetative growth and chemical constituents of rosemary cuttings. The application of auxin at $5 \mathrm{ppm}$ IBA positively affected rosemary rooting of cuttings by $92 \%$ (Gudeva et al., 2017). Vasca-Zamfir et al. (2018) evaluate the impact of rhizogenic biostimulator Clonex gel treatments on rooting of rosemary cuttings and found that, the biostimulator increased rooting percentage of cuttings and increasing of total chlorophyll and in the leaves. Varban et al. (2018) found that the percentage of rosemary rooting cuttings was higher by using the mixed substrate (peat + perlite) with or without Chryzotop $(0.25 \%$ beta-indole butyric acid. Bahedh (2020) found that parsley extract can be used as natural rooting promoter for stem cuttings of some plants as a new method instead of plant growth regulators regardless it's chemical composition (the chemical analysis of parsley extract showed the presence of IAA, $\mathrm{GA}_{3}$, cytokinin, highly activity of antioxidant enzymes and several amino acids, including Tryptophan, Glutamic acid, Alanine, Valine, Tyrosine, Phenyl Alanine, Histidine, Ornithine, Arginine and other amino acids especially in the concentration of $2.5 \mathrm{~g} / 1$ of parsley extract. The purpose of the research was to study the influence of the newly compound (Phos root $\mathrm{X}$ ) on rooting potential of rosemary cutting with or without auxin (IBA) to stimulate roots of rosemary cuttings.

\section{MATERIALS AND METHODS}

\section{Location and duration:}

The research was carried out during two successive seasons of 2019 and 2020 on $20^{\text {th }}$ of July for the first experiment, and on $25^{\text {th }}$ of October for the second one. Both experiments were conducted in the Ornamental Farm of the Horticulture Department, Faculty of Agriculture, Ain Shams University, Cairo, Egypt.

\section{Plant material preparation:}

Cuttings of rosemary stock mother plants in the ornamental farm about $15 \mathrm{~cm}$ in length for both terminal and medial type were used as plant materials.

\section{Culture procedures and treatments used:}

All cuttings were cultured in clay pots (25 $\mathrm{cm}$ in diameter) filled with sand:loam $(1: 1, \mathrm{v} / \mathrm{v})$, every pot contained three cuttings. The terminal cuttings were treated with the cutting rooting promotor by irrigating pots with the solutions at the tested concentrations after culture immediately. For the first experiments (only terminal cuttings) the cuttings were treated with Phos root- $\mathrm{X}$ as follows:

1. 0 level (control treatment).

2. $0.5 \mathrm{~cm}^{3} / 1$.

3. $1.0 \mathrm{~cm}^{3} / 1$.

4. $1.5 \mathrm{~cm}^{3} / 1$.

5. $2.0 \mathrm{~cm}^{3} / 1$.

6. $2.5 \mathrm{~cm}^{3} / 1$.

$7.3 \mathrm{~cm}^{3} / 1$. 
Chemical constituents of Phos root-X is shown in Table (1).

For the second experiment (terminal and medial cuttings were used) the treatments were as follows:

The terminal cuttings were treated with:

1. $0 \mathrm{ppm} \mathrm{IBA}+0 \mathrm{~cm}^{3} / 1$ of Phos root-x/pot.

2. $100 \mathrm{ppm} \mathrm{IBA}+0 \mathrm{~cm}^{3} / 1$ of Phos root-x/pot.

3. $500 \mathrm{ppm} \mathrm{IBA}+0 \mathrm{~cm}^{3} / 1$ of Phos root-x/pot.

4. $0 \mathrm{ppm} \mathrm{IBA}+1 \mathrm{~cm}^{3} / 1$ Phos root-x/pot.

5. $100 \mathrm{ppm} \mathrm{IBA+1} \mathrm{cm}^{3} / 1$ Phos root-x/pot.

6. $500 \mathrm{ppm} \mathrm{IBA}+1 \mathrm{~cm}^{3} / 1$ Phos root-x/pot.

7. $0 \mathrm{ppm} \mathrm{IBA}+2 \mathrm{~cm}^{3} / 1$ Phos root-x/pot.

8. $100 \mathrm{ppm} \mathrm{IBA+2} \mathrm{cm}^{3} / 1$ Phos root-x/pot.

9. $500 \mathrm{ppm} \mathrm{IBA}+2 \mathrm{~cm}^{3} / 1$ Phos root-x/pot.

10. $0 \mathrm{ppm} \mathrm{IBA}+3 \mathrm{~cm}^{3} / 1$ Phos root-x/pot.

11. $100 \mathrm{ppm} \mathrm{IBA}+3 \mathrm{~cm}^{3} / 1$ Phos root-x/pot.

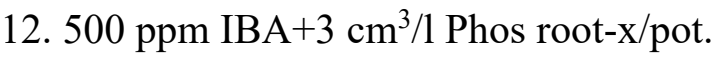

The same treatments as mentioned above were done for middle cuttings of rosemary plants.

\section{Data recorded and statistical analysis:}

Rooting \% of cuttings, survival \% of cuttings, dry matter $\%$, root number, root length $(\mathrm{cm})$ fresh and dry weights $(\mathrm{g}), \mathrm{C} / \mathrm{N}$ ratio were recorded. Data were collected every week for one month for the first experiment, after one month one time for the second experiment. Experiment was settled in a complete randomized design. Data was subjected to one-way analysis of variance as described by Snedecor and Cochran (1989). The means were compared according to Duncan test $(\mathrm{P} \leq 0.05)$ to evaluate differences among means (Duncan, 1955).

\section{RESULTS AND DISCUSSION}

\section{The first experiment:}

Data presented in Table (2) showed that, the dry matter \% increased with the development of rooting in the third and

Table 1. The constituents of Phos root-x.

\begin{tabular}{llllll}
\hline Components & Values & Components & Values & Components & Values \\
\hline Aspartic Acid & $1.52 \%$ & Leucine & $0.70 \%$ & Proline & $2.03 \%$ \\
Therionine & $1.06 \%$ & Lysine & $0.49 \%$ & Phenylalanine & $0.67 \%$ \\
Serine & $2.09 \%$ & Hustidine & $0.19 \%$ & Tyrosine & $0.22 \%$ \\
Glutamic Acid & $2.41 \%$ & Valine & $1.35 \%$ & Argenine & $1.50 \%$ \\
Glycine & $1.75 \%$ & Isoleucine & $0.62 \%$ & Alanine & $1.04 \%$ \\
$\mathbf{P}_{2} \mathbf{O}_{5}$ & $20 \%$ & $\mathbf{N}$ & $3 \%$ & $\mathbf{C u}$ & $0.3 \%$ \\
\hline
\end{tabular}

* (w/v) \% free Amino Acid Pram plant source.

Table 2. Influence of Phos root-x on dry matter \% of terminal cuttings of rosemary plants during rooting in two successive 2019 and 2020 seasons.

\begin{tabular}{|c|c|c|c|c|c|c|c|c|}
\hline \multirow{3}{*}{ Treatments } & \multicolumn{8}{|c|}{ Time } \\
\hline & \multicolumn{2}{|c|}{ First week } & \multicolumn{2}{|c|}{ Second week } & \multicolumn{2}{|c|}{ Third week } & \multicolumn{2}{|c|}{ Fourth week } \\
\hline & $1^{\text {st }}$ season & $2^{\text {nd }}$ season & $1^{\text {st }}$ season & $2^{\text {nd }}$ season & $1^{\text {st }}$ season & $2^{\text {nd }}$ season & $1^{\text {st }}$ season & $2^{\text {nd }}$ season \\
\hline 1 & $38.06 \mathrm{a}$ & $39.28 \mathrm{ab}$ & $45.12 \mathrm{bc}$ & $45.39 \mathrm{c}$ & $53.76 \mathrm{a}$ & $48.64 \mathrm{ab}$ & $50.96 \mathrm{a}$ & $46.21 \mathrm{~b}$ \\
\hline 2 & $39.67 \mathrm{a}$ & $45.75 \mathrm{a}$ & $49.80 \mathrm{~b}$ & $46.22 \mathrm{c}$ & $41.70 \mathrm{a}$ & $66.07 \mathrm{a}$ & $54.30 \mathrm{a}$ & $50.50 \mathrm{ab}$ \\
\hline 3 & $38.96 \mathrm{a}$ & $43.91 \mathrm{a}$ & $47.44 \mathrm{bc}$ & $52.65 \mathrm{ab}$ & $59.22 \mathrm{a}$ & $51.24 \mathrm{ab}$ & $63.72 \mathrm{a}$ & $55.08 \mathrm{ab}$ \\
\hline 4 & 33.49 bc & $31.30 \mathrm{c}$ & $56.63 \mathrm{a}$ & $49.62 \mathrm{bc}$ & $52.76 \mathrm{a}$ & $54.21 \mathrm{ab}$ & $53.91 \mathrm{a}$ & $50.45 \mathrm{ab}$ \\
\hline 5 & $31.38 \mathrm{c}$ & $33.36 \mathrm{bc}$ & $42.72 \mathrm{c}$ & $54.79 \mathrm{a}$ & $50.59 \mathrm{a}$ & $49.02 \mathrm{ab}$ & $47.48 \mathrm{a}$ & $50.06 \mathrm{ab}$ \\
\hline 6 & $36.83 \mathrm{ab}$ & $33.18 \mathrm{bc}$ & $41.75 \mathrm{c}$ & $52.59 \mathrm{ab}$ & $48.75 \mathrm{a}$ & $48.11 b$ & $45.66 \mathrm{a}$ & $48.09 \mathrm{~b}$ \\
\hline 7 & $32.26 \mathrm{c}$ & $32.08 \mathrm{bc}$ & $57.94 \mathrm{a}$ & $50.09 \mathrm{a}-\mathrm{c}$ & $53.55 \mathrm{a}$ & $54.77 \mathrm{ab}$ & $55,40 \mathrm{a}$ & $67.58 \mathrm{a}$ \\
\hline
\end{tabular}

Dissimilar letters designate the significance among means $(p<0.05)$ using Duncan's multiple range test. 
fourth harvest time, especially with 2 and 3 $\mathrm{cm}^{3} / 1$ of Phos root-x treatments.

Data in Table (3) showed that for all rooting parameters the best treatments were resulted from 2 and $2.5 \mathrm{~cm}^{3} / 1$ of Phos root-X (rooting promotor) compared to the other treatments. The data may be due to amino acids complex (Phos root-x new rooting promotor), these results were in harmony with Bahedh (2020) when using parsley extracts which contains some hormones and several amino acids.

\section{$\mathrm{C} / \mathrm{N}$ ratio:}

Data in Fig. (1) showed a decreasing of $\mathrm{C} / \mathrm{N}$ ratio with the increasing of Phos root-X concentration, this may be due to the increasing of total nitrogen with increasing of amino acids in Phos root-x amino acids complex.

\section{The second experiment:}

Dry matter of plantlet (\%):

Data in Table (4) showed that, the dry matter $\%$ in middle cuttings were better compared with terminal cuttings in both seasons of study. There were no significant differences between treatments inside both types of cuttings during first season, while in the second season, the highest value resulted from $100 \mathrm{ppm}$ IBA plus $3 \mathrm{~cm}^{3} / 1$ of Phos root-x inside middle cutting and 100 IBA

Table 3. Influence of Phos root-x treatments on rooting parameters of rosemary terminal cuttings during 2019 and 2020 seasons.

\begin{tabular}{|c|c|c|c|c|c|c|c|c|}
\hline \multirow{2}{*}{ Treatments } & \multicolumn{2}{|c|}{ Number of roots } & \multicolumn{2}{|c|}{ Length of roots $(\mathrm{cm})$} & \multicolumn{2}{|c|}{ Rooting percentage } & \multicolumn{2}{|c|}{ Survival percentage } \\
\hline & $1^{\text {st }}$ season & $2^{\text {nd }}$ season & $1^{\text {st }}$ season & $2^{\text {nd }}$ season & $1^{\text {st }}$ season & $2^{\text {nd }}$ season & $1^{\text {st }}$ season & $2^{\text {nd }}$ season \\
\hline 1 & $1.00 \mathrm{c}$ & $1.00 \mathrm{~cd}$ & $0.50 \mathrm{~b}$ & $0.50 \mathrm{c}$ & $10.00 \mathrm{~d}$ & $10.00 \mathrm{~d}$ & $70.00 \mathrm{c}$ & $70.00 \mathrm{c}$ \\
\hline 2 & $1.66 \mathrm{bc}$ & $1.66 \mathrm{~b}-\mathrm{d}$ & $0.50 \mathrm{~b}$ & $0.56 \mathrm{c}$ & $30.00 \mathrm{c}$ & $30.00 \mathrm{c}$ & $70.00 \mathrm{c}$ & $70.00 \mathrm{c}$ \\
\hline 3 & $2.00 \mathrm{bc}$ & $2.00 \mathrm{bc}$ & $0.55 \mathrm{~b}$ & $1.00 \mathrm{c}$ & $10.00 \mathrm{~d}$ & $10.00 \mathrm{~d}$ & $70.00 \mathrm{c}$ & $70.00 \mathrm{c}$ \\
\hline 4 & $4.33 \mathrm{ab}$ & $3.00 \mathrm{ab}$ & $1.16 \mathrm{~b}$ & $1.98 \mathrm{~b}$ & $60.00 \mathrm{~b}$ & $60.00 \mathrm{~b}$ & $80.00 \mathrm{~b}$ & $80.00 \mathrm{~b}$ \\
\hline 5 & $5.66 \mathrm{a}$ & $4.00 \mathrm{a}$ & $4.00 \mathrm{a}$ & $3.33 \mathrm{a}$ & $70.00 \mathrm{a}$ & $70.00 \mathrm{a}$ & $90.00 \mathrm{a}$ & $90.00 \mathrm{a}$ \\
\hline 6 & $6.66 \mathrm{a}$ & $4.00 \mathrm{a}$ & $2.63 \mathrm{a}$ & $3.40 \mathrm{a}$ & $70.00 \mathrm{a}$ & $70.00 \mathrm{a}$ & $80.00 \mathrm{~b}$ & $80.00 \mathrm{~b}$ \\
\hline 7 & $2.00 \mathrm{bc}$ & $0.34 \mathrm{~d}$ & $0.50 \mathrm{~b}$ & $0.28 \mathrm{c}$ & $10.00 \mathrm{~d}$ & $10.00 \mathrm{~d}$ & $90.00 \mathrm{a}$ & $90.00 \mathrm{a}$ \\
\hline
\end{tabular}

Dissimilar letters designate the significance among means $(p<0.05)$ using Duncan's multiple range test.

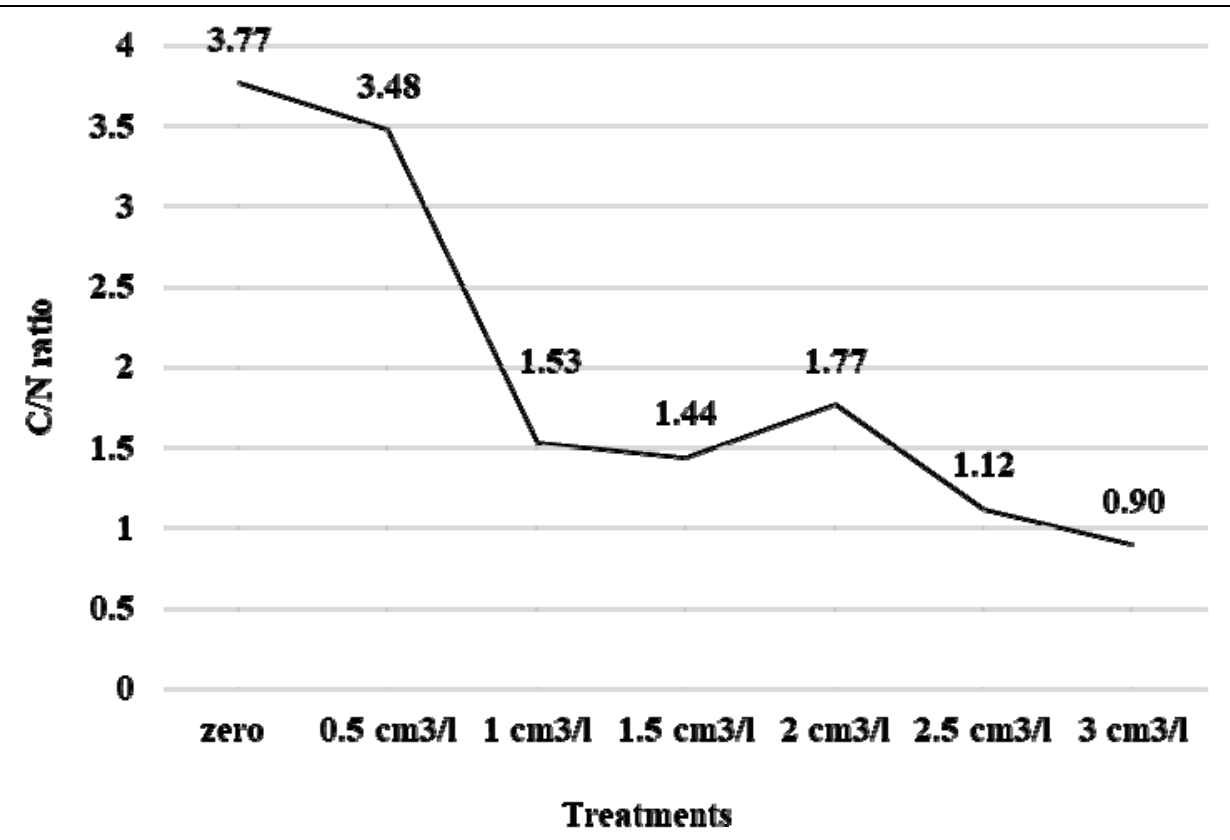

Fig. 1. Effect of Phos root-x treatments on $\mathrm{C} / \mathrm{N}$ ratio of rosemary terminal cuttings. 
Table 4. Influence of Phos root-x compound and/or IBA on dry matter \% of rosemary middle and terminal cuttings during 2019 and 2020 seasons.

\begin{tabular}{ccccccc}
\hline Treatments & Middle & $\begin{array}{c}\mathbf{1}^{\text {st }} \text { season } \\
\text { Terminal }\end{array}$ & Mean & Middle & $\begin{array}{c}\mathbf{2}^{\text {nd }} \text { season } \\
\text { Terminal }\end{array}$ & Mean \\
\hline $\mathbf{1}$ & $49.5 \mathrm{a}$ & $85.0 \mathrm{a}$ & $67.2 \mathrm{BC}$ & $54.3 \mathrm{c}-\mathrm{g}$ & $78.9 \mathrm{ab}$ & $66.6 \mathrm{~A}$ \\
$\mathbf{2}$ & $54.9 \mathrm{a}$ & $89.0 \mathrm{a}$ & $71.9 \mathrm{AB}$ & $52.3 \mathrm{e}-\mathrm{g}$ & $82.4 \mathrm{a}$ & $67.4 \mathrm{~A}$ \\
$\mathbf{3}$ & $52.2 \mathrm{a}$ & $70.4 \mathrm{a}$ & $61.3 \mathrm{BC}$ & $57.6 \mathrm{c}-\mathrm{g}$ & $54.8 \mathrm{c}-\mathrm{g}$ & $56.2 \mathrm{~B}-\mathrm{D}$ \\
$\mathbf{4}$ & $47.0 \mathrm{a}$ & $56.3 \mathrm{a}$ & $51.7 \mathrm{C}$ & $48.6 \mathrm{fg}$ & $55.8 \mathrm{c}-\mathrm{g}$ & $52.2 \mathrm{CD}$ \\
$\mathbf{5}$ & $43.3 \mathrm{a}$ & $62.6 \mathrm{a}$ & $53.0 \mathrm{C}$ & $46.5 \mathrm{~g}$ & $54.9 \mathrm{c}-\mathrm{g}$ & $50.7 \mathrm{D}$ \\
$\mathbf{6}$ & $57.1 \mathrm{a}$ & $66.0 \mathrm{a}$ & $61.5 \mathrm{BC}$ & $54.4 \mathrm{c}-\mathrm{g}$ & $66.4 \mathrm{~b}-\mathrm{d}$ & $60.4 \mathrm{~A}-\mathrm{C}$ \\
$\mathbf{7}$ & $55.5 \mathrm{a}$ & $56.5 \mathrm{a}$ & $56.0 \mathrm{BC}$ & $58.2 \mathrm{c}-\mathrm{g}$ & $54.0 \mathrm{c}-\mathrm{g}$ & $56.1 \mathrm{~B}-\mathrm{D}$ \\
$\mathbf{8}$ & $74.5 \mathrm{a}$ & $101.8 \mathrm{a}$ & $88.1 \mathrm{~A}$ & $63.8 \mathrm{c}-\mathrm{e}$ & $54.0 \mathrm{c}-\mathrm{g}$ & $58.9 \mathrm{~A}-\mathrm{D}$ \\
$\mathbf{9}$ & $63.0 \mathrm{a}$ & $66.7 \mathrm{a}$ & $64.9 \mathrm{BC}$ & $60.5 \mathrm{c}-\mathrm{f}$ & $53.3 \mathrm{~d}-\mathrm{g}$ & $56.9 \mathrm{~B}-\mathrm{D}$ \\
$\mathbf{1 0}$ & $61.1 \mathrm{a}$ & $55.6 \mathrm{a}$ & $58.3 \mathrm{BC}$ & $60.0 \mathrm{c}-\mathrm{f}$ & $49.2 \mathrm{fg}$ & $54.6 \mathrm{CD}$ \\
$\mathbf{1 1}$ & $58.1 \mathrm{a}$ & $79.6 \mathrm{a}$ & $68.9 \mathrm{BC}$ & $66.9 \mathrm{bc}$ & $53.1 \mathrm{e}-\mathrm{g}$ & $60.0 \mathrm{~A}-\mathrm{C}$ \\
$\mathbf{1 2}$ & $69.4 \mathrm{a}$ & $54.1 \mathrm{a}$ & $61.8 \mathrm{BC}$ & $63.3 \mathrm{c}-\mathrm{e}$ & $65.4 \mathrm{c}-\mathrm{e}$ & $64.4 \mathrm{AB}$ \\
Mean & $57.1 \mathrm{~B}$ & $70.3 \mathrm{~A}$ & & $57.2 \mathrm{~A}$ & $60.2 \mathrm{~A}$ & \\
\hline
\end{tabular}

Dissimilar letters designate the significance among means $(p<0.05)$ using Duncan's multiple range test.

only inside terminal cuttings. For the interaction between the two type of cuttings, the highest value was resulted with $100 \mathrm{ppm}$ IBA only.

\section{Root number of plantlets:}

Data listed in Table (5) showed that the best type of cutting was the terminal for both seasons of the research. The highest value of root number was obtained with $3 \mathrm{~cm}^{3} / 1$ of Phos-root-x without IBA in middle cutting during first and second seasons and $3 \mathrm{~cm}^{3} / 1$ of Phos root-x without IBA and $3 \mathrm{~cm}^{3} / 1$ with 500 IBA inside terminal cuttings during first and second seasons of the research. For the interaction, the best treatment was $3 \mathrm{~cm}^{3} / 1$ Phos root-x.

\section{Root length of plantlet:}

Data presented in Table (6) showed that for type of cuttings, terminal cuttings gave better values than middle ones. For both middle and terminal types of cuttings higher value with $3 \mathrm{~cm}^{3} / 1$ of Phos root-x plus 100 ppm IBA during the two seasons. A higher value obtained with treatment $3 \mathrm{~cm}^{3} / 1$ of Phos root-x without IBA.

\section{Root fresh and dry weight of plantlet:}

Data in Tables (7 and 8) showed that the best type of cuttings for fresh and dry weights of roots was the terminal cuttings. For middle type of cutting Phos root-x at 3 $\mathrm{cm}^{3} / 1$ plus $100 \mathrm{ppm}$ of IBA gave the highest values in the two seasons of the research, while for terminal type, the highest values resulted from $3 \mathrm{~cm}^{3} / 1$ of Phos root-x plus 500 ppm IBA.

\section{Survival percentage:}

Data presented in Table (9) showed that, the terminal cuttings were better than middle ones in survival $\%$ and treatment $3 \mathrm{~cm}^{3} / 1$ Phos root-x plus 500 ppm IBA gave the highest value.

\section{Rooting percentage:}

Data listed in Table (10) showed that the highest rooting values were obtained with terminal cuttings than the middle one's type. 
Table 5. Influence of Phos root-x compound and/or IBA on number of roots of rosemary middle and terminal cuttings during 2019 and 2020 seasons.

\begin{tabular}{ccccccc}
\hline Treatments & Middle & $\begin{array}{c}\mathbf{1}^{\text {st }} \text { season } \\
\text { Terminal }\end{array}$ & Mean & Middle & $\begin{array}{c}\mathbf{2}^{\text {nd }} \text { season } \\
\text { Terminal }\end{array}$ & Mean \\
\hline $\mathbf{1}$ & $1.00 \mathrm{i}$ & $1.00 \mathrm{i}$ & $1.00 \mathrm{D}$ & $1.13 \mathrm{i}$ & $1.13 \mathrm{i}$ & $1.13 \mathrm{D}$ \\
$\mathbf{2}$ & $1.00 \mathrm{i}$ & $1.00 \mathrm{i}$ & $1.00 \mathrm{D}$ & $1.13 \mathrm{i}$ & $1.13 \mathrm{i}$ & $1.13 \mathrm{D}$ \\
$\mathbf{3}$ & $1.00 \mathrm{i}$ & $7.33 \mathrm{a}-\mathrm{c}$ & $4.17 \mathrm{BC}$ & $1.13 \mathrm{i}$ & $8.31 \mathrm{a}-\mathrm{c}$ & $4.72 \mathrm{BC}$ \\
$\mathbf{4}$ & $3.00 \mathrm{f}-\mathrm{i}$ & $5.33 \mathrm{c}-\mathrm{e}$ & $4.17 \mathrm{BC}$ & $3.40 \mathrm{f}-\mathrm{i}$ & $6.04 \mathrm{c}-\mathrm{e}$ & $4.72 \mathrm{BC}$ \\
$\mathbf{5}$ & $1.00 \mathrm{i}$ & $7.67 \mathrm{ab}$ & $4.33 \mathrm{BC}$ & $1.13 \mathrm{i}$ & $8.69 \mathrm{ab}$ & $4.91 \mathrm{BC}$ \\
$\mathbf{6}$ & $1.00 \mathrm{i}$ & $9.00 \mathrm{a}$ & $5.00 \mathrm{~B}$ & $1.13 \mathrm{i}$ & $10.20 \mathrm{a}$ & $5.67 \mathrm{~B}$ \\
$\mathbf{7}$ & $4.00 \mathrm{~d}-\mathrm{g}$ & $6.00 \mathrm{~b}-\mathrm{d}$ & $5.00 \mathrm{~B}$ & $4.53 \mathrm{~d}-\mathrm{g}$ & $6.80 \mathrm{~b}-\mathrm{d}$ & $5.67 \mathrm{~B}$ \\
$\mathbf{8}$ & $1.00 \mathrm{i}$ & $1.67 \mathrm{~g}-\mathrm{i}$ & $1.33 \mathrm{D}$ & $1.13 \mathrm{i}$ & $1.89 \mathrm{hi}$ & $1.51 \mathrm{D}$ \\
$\mathbf{9}$ & $2.00 \mathrm{~g}-\mathrm{i}$ & $4.00 \mathrm{~d}-\mathrm{g}$ & $3.00 \mathrm{C}$ & $2.27 \mathrm{~g}-\mathrm{i}$ & $4.53 \mathrm{~d}-\mathrm{g}$ & $3.40 \mathrm{C}$ \\
$\mathbf{1 0}$ & $5.33 \mathrm{c}-\mathrm{e}$ & $9.00 \mathrm{a}$ & $7.17 \mathrm{~A}$ & $6.04 \mathrm{c}-\mathrm{e}$ & $10.20 \mathrm{a}$ & $8.12 \mathrm{~A}$ \\
$\mathbf{1 1}$ & $4.33 \mathrm{~d}-\mathrm{f}$ & $3.33 \mathrm{e}-\mathrm{h}$ & $3.83 \mathrm{BC}$ & $4.91 \mathrm{~d}-\mathrm{f}$ & $3.78 \mathrm{e}-\mathrm{h}$ & $4.34 \mathrm{BC}$ \\
$\mathbf{1 2}$ & $1.33 \mathrm{hi}$ & $9.00 \mathrm{a}$ & $5.17 \mathrm{~B}$ & $1.51 \mathrm{hi}$ & $10.20 \mathrm{a}$ & $5.85 \mathrm{C}$ \\
Mean & $2.17 \mathrm{~B}$ & $5.36 \mathrm{~A}$ & & $2.45 \mathrm{~B}$ & $6.07 \mathrm{~A}$ & \\
\hline
\end{tabular}

Dissimilar letters designate the significance among means $(p<0.05)$ using Duncan's multiple range test.

Table 6. Influence of Phos root-x compound and/or IBA on root length of rosemary middle and terminal cuttings during 2019 and 2020 seasons.

\begin{tabular}{ccccccc}
\hline Treatments & Middle & $\begin{array}{c}\mathbf{1}^{\text {st }} \text { season } \\
\text { Terminal }\end{array}$ & Mean & Middle & $\begin{array}{c}\mathbf{2}^{\text {nd }} \text { season } \\
\text { Terminal }\end{array}$ & Mean \\
\hline $\mathbf{1}$ & $0.50 \mathrm{fg}$ & $0.50 \mathrm{fg}$ & $0.50 \mathrm{E}$ & $0.57 \mathrm{fg}$ & $0.57 \mathrm{fg}$ & $0.57 \mathrm{E}$ \\
$\mathbf{2}$ & $0.50 \mathrm{fg}$ & $0.30 \mathrm{~g}$ & $0.40 \mathrm{E}$ & $0.57 \mathrm{fg}$ & $0.34 \mathrm{~g}$ & $0.45 \mathrm{E}$ \\
$\mathbf{3}$ & $0.50 \mathrm{fg}$ & $3.00 \mathrm{~b}$ & $1.75 \mathrm{CD}$ & $0.57 \mathrm{fg}$ & $3.40 \mathrm{~b}$ & $1.98 \mathrm{CD}$ \\
$\mathbf{4}$ & $1.03 \mathrm{ef}$ & $2.60 \mathrm{bc}$ & $1.82 \mathrm{CD}$ & $1.17 \mathrm{ef}$ & $2.95 \mathrm{bc}$ & $2.06 \mathrm{CD}$ \\
$\mathbf{5}$ & $0.50 \mathrm{fg}$ & $3.00 \mathrm{~b}$ & $1.75 \mathrm{CD}$ & $0.57 \mathrm{fg}$ & $3.40 \mathrm{~b}$ & $1.98 \mathrm{CD}$ \\
$\mathbf{6}$ & $0.50 \mathrm{fg}$ & $3.67 \mathrm{a}$ & $2.08 \mathrm{BC}$ & $0.57 \mathrm{fg}$ & $4.15 \mathrm{a}$ & $2.36 \mathrm{BC}$ \\
$\mathbf{7}$ & $1.50 \mathrm{de}$ & $3.00 \mathrm{~b}$ & $2.25 \mathrm{~B}$ & $1.70 \mathrm{de}$ & $3.40 \mathrm{~b}$ & $2.55 \mathrm{~B}$ \\
$\mathbf{8}$ & $0.50 \mathrm{fg}$ & $0.87 \mathrm{fg}$ & $0.68 \mathrm{E}$ & $0.57 \mathrm{fg}$ & $0.98 \mathrm{fg}$ & $0.77 \mathrm{E}$ \\
$\mathbf{9}$ & $1.00 \mathrm{ef}$ & $2.07 \mathrm{~cd}$ & $1.53 \mathrm{~B}$ & $1.13 \mathrm{ef}$ & $2.34 \mathrm{~cd}$ & $1.74 \mathrm{D}$ \\
$\mathbf{1 0}$ & $2.53 \mathrm{bc}$ & $4.20 \mathrm{a}$ & $3.37 \mathrm{~A}$ & $2.87 \mathrm{bc}$ & $4.76 \mathrm{a}$ & $3.81 \mathrm{~A}$ \\
$\mathbf{1 1}$ & $2.23 \mathrm{c}$ & $1.00 \mathrm{fg}$ & $1.62 \mathrm{D}$ & $2.53 \mathrm{c}$ & $1.13 \mathrm{ef}$ & $1.83 \mathrm{D}$ \\
$\mathbf{1 2}$ & $0.83 \mathrm{fg}$ & $2.87 \mathrm{~b}$ & $1.85 \mathrm{~B}-\mathrm{D}$ & $0.94 \mathrm{fg}$ & $3.25 \mathrm{fg}$ & $2.10 \mathrm{~B}-\mathrm{D}$ \\
Mean & $1.01 \mathrm{~B}$ & $2.26 \mathrm{~A}$ & & $1.15 \mathrm{~B}$ & $2.56 \mathrm{~A}$ & \\
\hline
\end{tabular}

Dissimilar letters designate the significance among means $(p<0.05)$ using Duncan's multiple range test. 
Table 7. Influence of Phos root-x compound and/or IBA on root fresh weight of rosemary middle and terminal cuttings during 2019 and 2020 seasons.

\begin{tabular}{|c|c|c|c|c|c|c|}
\hline \multirow{2}{*}{ Treatments } & \multicolumn{3}{|c|}{$1^{\text {st }}$ season } & \multicolumn{3}{|c|}{$2^{\text {nd }}$ season } \\
\hline & Middle & Terminal & Mean & Middle & Terminal & Mean \\
\hline 1 & $0.100 \mathrm{e}$ & $0.100 \mathrm{e}$ & $0.100 \mathrm{~B}$ & $0.113 \mathrm{e}$ & $0.113 \mathrm{e}$ & $0.113 \mathrm{~B}$ \\
\hline 2 & $0.100 \mathrm{e}$ & $0.097 \mathrm{e}$ & $0.098 \mathrm{~B}$ & $0.113 \mathrm{e}$ & $0.110 \mathrm{e}$ & $0.111 \mathrm{~B}$ \\
\hline 3 & $0.100 \mathrm{e}$ & $0.793 \mathrm{ab}$ & $0.447 \mathrm{~A}$ & $0.113 \mathrm{e}$ & $0.899 \mathrm{ab}$ & $0.506 \mathrm{~A}$ \\
\hline 4 & $0.310 \mathrm{de}$ & $0.630 \mathrm{~b}-\mathrm{d}$ & $0.470 \mathrm{~A}$ & $0.351 \mathrm{de}$ & $0.714 \mathrm{~b}-\mathrm{d}$ & $0.533 \mathrm{~A}$ \\
\hline 5 & $0.100 \mathrm{e}$ & $0.913 \mathrm{ab}$ & $0.507 \mathrm{~A}$ & $0.113 \mathrm{e}$ & $1.035 \mathrm{a}$ & $0.574 \mathrm{~A}$ \\
\hline 6 & $0.140 \mathrm{e}$ & $1.007 \mathrm{a}$ & $0.573 \mathrm{~A}$ & $0.159 \mathrm{e}$ & $1.141 \mathrm{a}$ & $0.650 \mathrm{~A}$ \\
\hline 7 & $0.410 \mathrm{c}-\mathrm{e}$ & $0.833 \mathrm{ab}$ & $0.622 \mathrm{~A}$ & $0.465 \mathrm{c}-\mathrm{e}$ & $0.944 \mathrm{ab}$ & $0.704 \mathrm{~A}$ \\
\hline 8 & $0.100 \mathrm{e}$ & $0.123 \mathrm{e}$ & $0.112 \mathrm{~B}$ & $0.113 \mathrm{e}$ & $0.140 \mathrm{e}$ & $0.127 \mathrm{~B}$ \\
\hline 9 & $0.200 \mathrm{e}$ & $0.620 \mathrm{~b}-\mathrm{d}$ & $0.410 \mathrm{~A}$ & $0.227 \mathrm{e}$ & $0.702 \mathrm{~b}-\mathrm{d}$ & $0.465 \mathrm{~A}$ \\
\hline 10 & $0.277 \mathrm{e}$ & $0.950 \mathrm{ab}$ & $0.613 \mathrm{~A}$ & $0.313 \mathrm{e}$ & $1.076 \mathrm{ab}$ & $0.695 \mathrm{~A}$ \\
\hline 11 & $0.693 \mathrm{a}-\mathrm{c}$ & $0.180 \mathrm{e}$ & $0.437 \mathrm{~A}$ & 0.786 a-c & $0.204 \mathrm{e}$ & $0.495 \mathrm{~A}$ \\
\hline 12 & $0.167 \mathrm{e}$ & $0.983 \mathrm{a}$ & $0.575 \mathrm{~A}$ & $0.189 \mathrm{e}$ & $1.114 \mathrm{a}$ & $0.651 \mathrm{~A}$ \\
\hline Mean & $0.225 \mathrm{~B}$ & $0.603 \mathrm{~A}$ & & $0.255 \mathrm{~B}$ & $0.683 \mathrm{~A}$ & \\
\hline
\end{tabular}

Dissimilar letters designate the significance among means $(p<0.05)$ using Duncan's multiple range test.

Table 8. Influence of Phos root-x compound and/or IBA on root dry weight of rosemary middle and terminal cuttings during 2019 and 2020 seasons.

\begin{tabular}{ccccccc}
\hline Treatments & Middle & $\begin{array}{c}\mathbf{1}^{\text {st }} \text { season } \\
\text { Terminal }\end{array}$ & Mean & Middle & $\begin{array}{c}\mathbf{2}^{\text {nd }} \text { season } \\
\text { Terminal }\end{array}$ & Mean \\
\hline $\mathbf{1}$ & $0.05 \mathrm{f}$ & $0.05 \mathrm{f}$ & $0.05 \mathrm{BC}$ & $0.06 \mathrm{f}$ & $0.06 \mathrm{f}$ & $0.06 \mathrm{C}$ \\
$\mathbf{2}$ & $0.05 \mathrm{f}$ & $0.05 \mathrm{f}$ & $0.05 \mathrm{BC}$ & $0.06 \mathrm{f}$ & $0.05 \mathrm{f}$ & $0.05 \mathrm{C}$ \\
$\mathbf{3}$ & $0.05 \mathrm{f}$ & $0.43 \mathrm{a}-\mathrm{c}$ & $0.24 \mathrm{~A}$ & $0.06 \mathrm{f}$ & $0.49 \mathrm{a}$ & $0.27 \mathrm{~A}$ \\
$\mathbf{4}$ & $0.19 \mathrm{c}-\mathrm{f}$ & $0.38 \mathrm{a}-\mathrm{d}$ & $0.28 \mathrm{~A}$ & $0.22 \mathrm{e}$ & $0.43 \mathrm{a}$ & $0.32 \mathrm{~A}$ \\
$\mathbf{5}$ & $0.05 \mathrm{f}$ & $0.44 \mathrm{ab}$ & $0.24 \mathrm{~A}$ & $0.06 \mathrm{f}$ & $0.49 \mathrm{a}$ & $0.28 \mathrm{~A}$ \\
$\mathbf{6}$ & $0.05 \mathrm{f}$ & $0.46 \mathrm{a}$ & $0.26 \mathrm{~A}$ & $0.06 \mathrm{f}$ & $0.52 \mathrm{a}$ & $0.29 \mathrm{~A}$ \\
$\mathbf{7}$ & $0.20 \mathrm{~b}-\mathrm{f}$ & $0.35 \mathrm{a}-\mathrm{d}$ & $0.27 \mathrm{~A}$ & $0.23 \mathrm{e}$ & $0.39 \mathrm{~d}$ & $0.31 \mathrm{~A}$ \\
$\mathbf{8}$ & $0.05 \mathrm{f}$ & $0.03 \mathrm{f}$ & $0.04 \mathrm{C}$ & $0.06 \mathrm{f}$ & $0.04 \mathrm{f}$ & $0.05 \mathrm{C}$ \\
$\mathbf{9}$ & $0.10 \mathrm{ef}$ & $0.34 \mathrm{a}-\mathrm{e}$ & $0.22 \mathrm{AB}$ & $0.11 \mathrm{e}$ & $0.39 \mathrm{~d}$ & $0.25 \mathrm{AB}$ \\
$\mathbf{1 0}$ & $0.14 \mathrm{~d}-\mathrm{f}$ & $0.52 \mathrm{a}$ & $0.33 \mathrm{~A}$ & $0.16 \mathrm{e}$ & $0.59 \mathrm{a}$ & $0.37 \mathrm{~A}$ \\
$\mathbf{1 1}$ & $0.39 \mathrm{a}-\mathrm{c}$ & $0.01 \mathrm{f}$ & $0.20 \mathrm{~A}-\mathrm{C}$ & $0.45 \mathrm{a}$ & $0.01 \mathrm{~g}$ & $0.23 \mathrm{AB}$ \\
$\mathbf{1 2}$ & $0.08 \mathrm{f}$ & $0.55 \mathrm{a}$ & $0.32 \mathrm{~A}$ & $0.09 \mathrm{f}$ & $0.62 \mathrm{a}$ & $0.36 \mathrm{~A}$ \\
Mean & $0.12 \mathrm{~B}$ & $0.30 \mathrm{~A}$ & & $0.13 \mathrm{~B}$ & $0.34 \mathrm{~A}$ & \\
\hline
\end{tabular}

Dissimilar letters designate the significance among means $(p<0.05)$ using Duncan's multiple range test. 
Table 9. Influence of Phos root-x compound and/or IBA on survival percentage of rosemary middle and terminal cuttings during 2019 and 2020 seasons.

\begin{tabular}{ccccccc}
\hline Treatments & Middle & $\begin{array}{c}\mathbf{1}^{\text {st }} \text { season } \\
\text { Terminal }\end{array}$ & Mean & Middle & $\begin{array}{c}\mathbf{2}^{\text {nd }} \text { season } \\
\text { Terminal }\end{array}$ & Mean \\
\hline $\mathbf{1}$ & $71.0 \mathrm{e}$ & $64.0 \mathrm{f}$ & $67.5 \mathrm{H}$ & $62.0 \mathrm{f}$ & $52.0 \mathrm{~g}$ & $57.0 \mathrm{I}$ \\
$\mathbf{2}$ & $42.7 \mathrm{~h}$ & $52.7 \mathrm{~g}$ & $47.7 \mathrm{~J}$ & $52.0 \mathrm{~g}$ & $82.0 \mathrm{~d}$ & $67.0 \mathrm{G}$ \\
$\mathbf{3}$ & $32.0 \mathrm{i}$ & $71.0 \mathrm{e}$ & $51.5 \mathrm{I}$ & $12.0 \mathrm{~h}$ & $92.0 \mathrm{c}$ & $52.0 \mathrm{~J}$ \\
$\mathbf{4}$ & $93.7 \mathrm{~b}$ & $93.0 \mathrm{bc}$ & $93.3 \mathrm{~B}$ & $72.0 \mathrm{e}$ & $100.0 \mathrm{a}$ & $86.0 \mathrm{C}$ \\
$\mathbf{5}$ & $83.0 \mathrm{~d}$ & $92.0 \mathrm{bc}$ & $87.5 \mathrm{D}$ & $62.0 \mathrm{f}$ & $62.0 \mathrm{f}$ & $62.0 \mathrm{H}$ \\
$\mathbf{6}$ & $21.3 \mathrm{j}$ & $82.0 \mathrm{~d}$ & $51.7 \mathrm{I}$ & $62.0 \mathrm{f}$ & $98.0 \mathrm{~b}$ & $80.0 \mathrm{E}$ \\
$\mathbf{7}$ & $98.0 \mathrm{a}$ & $98.0 \mathrm{a}$ & $98.0 \mathrm{~A}$ & $82.0 \mathrm{~d}$ & $98.0 \mathrm{~b}$ & $90.0 \mathrm{~B}$ \\
$\mathbf{8}$ & $41.0 \mathrm{~h}$ & $33.0 \mathrm{i}$ & $37.0 \mathrm{~K}$ & $62.0 \mathrm{f}$ & $98.0 \mathrm{~b}$ & $80.0 \mathrm{E}$ \\
$\mathbf{9}$ & $71.0 \mathrm{e}$ & $98.0 \mathrm{a}$ & $84.5 \mathrm{E}$ & $62.0 \mathrm{f}$ & $98.0 \mathrm{~b}$ & $80.0 \mathrm{E}$ \\
$\mathbf{1 0}$ & $83.0 \mathrm{~d}$ & $98.0 \mathrm{a}$ & $90.5 \mathrm{C}$ & $72.0 \mathrm{e}$ & $98.0 \mathrm{~b}$ & $85.0 \mathrm{D}$ \\
$\mathbf{1 1}$ & $83.0 \mathrm{~d}$ & $71.3 \mathrm{e}$ & $77.2 \mathrm{G}$ & $52.0 \mathrm{~g}$ & $92.0 \mathrm{c}$ & $72.0 \mathrm{~F}$ \\
$\mathbf{1 2}$ & $71.0 \mathrm{e}$ & $91.0 \mathrm{c}$ & $81.0 \mathrm{~F}$ & $100.0 \mathrm{a}$ & $98.0 \mathrm{~b}$ & $99.0 \mathrm{~A}$ \\
Mean & $65.9 \mathrm{~B}$ & $78.7 \mathrm{~A}$ & & $62.7 \mathrm{~B}$ & $89.0 \mathrm{~A}$ & \\
\hline
\end{tabular}

Dissimilar letters designate the significance among means $(p<0.05)$ using Duncan's multiple range test.

Table 10. Influence of Phos root-x compound and/or IBA on rooting percentage of rosemary middle and terminal cuttings during 2019 and 2020 seasons.

\begin{tabular}{ccccccc}
\hline Treatments & Middle & $\begin{array}{c}\mathbf{1}^{\text {st }} \text { season } \\
\text { Terminal }\end{array}$ & Mean & Middle & $\begin{array}{c}\mathbf{2}^{\text {nd }} \text { season } \\
\text { Terminal }\end{array}$ & Mean \\
\hline $\mathbf{1}$ & $32.7 \mathrm{f}$ & $12.3 \mathrm{~h}$ & $22.5 \mathrm{E}$ & $42.0 \mathrm{~g}$ & $12.0 \mathrm{i}$ & $27.0 \mathrm{G}$ \\
$\mathbf{2}$ & $12.3 \mathrm{~h}$ & $22.0 \mathrm{~g}$ & $17.2 \mathrm{~F}$ & $12.0 \mathrm{i}$ & $12.0 \mathrm{i}$ & $12.0 \mathrm{I}$ \\
$\mathbf{3}$ & $12.3 \mathrm{~h}$ & $72.7 \mathrm{~b}$ & $42.5 \mathrm{C}$ & $12.0 \mathrm{i}$ & $82.0 \mathrm{c}$ & $47.0 \mathrm{C}$ \\
$\mathbf{4}$ & $22.3 \mathrm{~g}$ & $72.7 \mathrm{~b}$ & $47.5 \mathrm{~B}$ & $12.0 \mathrm{i}$ & $82.0 \mathrm{c}$ & $47.0 \mathrm{C}$ \\
$\mathbf{5}$ & $12.3 \mathrm{~h}$ & $82.7 \mathrm{a}$ & $47.5 \mathrm{~B}$ & $12.0 \mathrm{i}$ & $22.0 \mathrm{~h}$ & $17.0 \mathrm{H}$ \\
$\mathbf{6}$ & $12.3 \mathrm{~h}$ & $61.3 \mathrm{c}$ & $36.8 \mathrm{D}$ & $12.0 \mathrm{i}$ & $52.0 \mathrm{f}$ & $32.0 \mathrm{~F}$ \\
$\mathbf{7}$ & $32.7 \mathrm{f}$ & $51.7 \mathrm{~d}$ & $42.2 \mathrm{C}$ & $12.0 \mathrm{i}$ & $72.0 \mathrm{~d}$ & $42.0 \mathrm{D}$ \\
$\mathbf{8}$ & $12.3 \mathrm{~h}$ & $32.7 \mathrm{f}$ & $22.5 \mathrm{E}$ & $12.0 \mathrm{i}$ & $62.0 \mathrm{e}$ & $37.0 \mathrm{E}$ \\
$\mathbf{9}$ & $12.3 \mathrm{~h}$ & $32.7 \mathrm{f}$ & $22.5 \mathrm{E}$ & $12.0 \mathrm{i}$ & $62.0 \mathrm{e}$ & $37.0 \mathrm{E}$ \\
$\mathbf{1 0}$ & $41.0 \mathrm{e}$ & $61.3 \mathrm{c}$ & $51.2 \mathrm{~A}$ & $12.0 \mathrm{i}$ & $92.0 \mathrm{~b}$ & $52.0 \mathrm{~B}$ \\
$\mathbf{1 1}$ & $51.7 \mathrm{~d}$ & $32.7 \mathrm{f}$ & $42.2 \mathrm{C}$ & $12.0 \mathrm{i}$ & $98.0 \mathrm{a}$ & $55.0 \mathrm{~A}$ \\
$\mathbf{1 2}$ & $22.0 \mathrm{~g}$ & $82.7 \mathrm{a}$ & $52.3 \mathrm{~A}$ & $12.0 \mathrm{i}$ & $62.0 \mathrm{e}$ & $37.0 \mathrm{E}$ \\
Mean & $23.0 \mathrm{~B}$ & $51.4 \mathrm{~A}$ & & $14.5 \mathrm{~B}$ & $59.2 \mathrm{~A}$ & \\
\hline
\end{tabular}

Dissimilar letters designate the significance among means $(p<0.05)$ using Duncan's multiple range test. 
A higher rooting $\%$ values obtained with Phos root-x $3 \mathrm{~cm}^{3} / 1$ plus 100 ppm of IBA.

It could be concluded that for all rooting parameter, the best types for rooting was the terminal type, and the higher concentrations of Phos root-x favored for better results especially with $3 \mathrm{~cm}^{3} / 1$ level. These results may be due to the higher concentrations of amino acids inside the new rooting promoter Phos root-x, these results were in harmony with the results obtained with Bahedh (2020) when using parsley extracts as a new growth regulator for rooting of the rosemary cuttings. It could be concluded that for all rooting parameter, the best types for rooting was the terminal type, this may be due to internal plant hormones such as auxin and other cofactors which are concentrated on plant apex where meristematic cells abound (Hartmann et al., 2002) and were more actively dividing cells which could be easily influenced by the hormonal treatment (Raji and Mohamed, 2012). The higher concentrations of Phoos root-x favored for better results especially with $3 \mathrm{~cm}^{3} / 1$ level, these results may be due to the higher concentrations of amino acids inside the new rooting promoter Phos root-x. The results obtained were in harmony with the results obtained by (Bahedh, 2020) when using parsley extracts as a new growth regulator for rooting of the rosemary cuttings, the plant extract can be used as natural rooting promoter for stem cuttings of some plants as a new method instead of plant growth regulators regardless it's chemical composition (the chemical analysis of parsley extract showed the presence of IAA, $\mathrm{GA}_{3}$, cytokinin, highly activity of antioxidant enzymes and several amino acids, including tryptophan, glutamic acid, alanine, valine, tyrosine, phenyl alanine, histidine, ornithine, arginine and other amino acids) especially in the concentration of 2.5 $\mathrm{g} / \mathrm{l}$ of parsley extract. These compounds enhance uptake of macro and microelements and their translocation within plants also increase the respiration rate and root growth, photosynthesis, and other metabolic processes (Dunsin et al., 2016).

\section{REFERENCES}

Bahedh, S.B. and Al Habib, A.A.S. (2020). Evaluation the activity of petroselinum rooting for stem cuttings of some plants. Al-Mustansiriyah Journal of Science, 31(1):22-30.

Bozin, B.; Mimica-Dukic, N.; Sanojlik, I. and Jovin, E. (2007). Antimicrobial and antioxidants properties of rosemary and sage (Rosmarinus officinalis L. and Salvia officinalis L., Lamiaceae) essential oils. J. Agric. Food Chem., 55:78797885 .

Chakaravati, S.; and Raghuvanshi, S.S. (2005). Rosemary (Rosmarinus officinalis): a useful medicinal herb. Vaniki-Sandesh, 29:26-27.

Duke, J.A. (2001). Handbook of Medical Herbs. Boca Raton, FL: CRC Prees, 677 p.

Duncan, D.B. (1955). Multiple range and multiple (F) test. Biometries, 11: 1-24.

Dunsin, O.; Ajiboye, G. and Adeyemo, T. (2016). Effect of alternative hormones on the rootability of Parkia biglobosa. Sci. Agr., 13(2):113-118.

Elhaak, M.A.; Matter, M.Z.; Zayed, M.A. and Gad, D.A. (2015). Propagation principles in using indole 3- butyric acid for rooting rosemary stem cuttings. Journal of Horticulture, 2(1):1-13. http://dx.doi.org/10.4172/2376-0354

Grieve, M. (1984). A Modern Herbal. Pengium, UK, $1056 \mathrm{p}$.

Gudeva, 1.K.; Trajkovic, F.; Mihajlov, L. and Troicki. J. (2017). Influence of different auxins on rooting of rosemary; sage and elderberry. Annual Research and Review in Biology, 12(5):1-8.

Hartmann, H.T.; Kester, D.E.; Davies, F.T. and Geneve, R.L. (2002). Plant Propagation Principles and Practices, $7^{\text {th }}$ Edition. Prentice Hall. New Jersey, USA, $880 \mathrm{p}$.

Karimi, M.; Berrichi, A. and Boukroute, A. (2014). Study of vegetative propagation 


\section{F.F. Lasheen et al.}

by cuttings of Thymus satureiodes. J. Mater. Environ. Sci., 5(4):1320-1325.

Massoud, H.Y. M.; Abd El-Baset, M.M. and Ghozzy, A. (2017). Effect of some natural products as an alternative chemical growth regulator on rooting response, growth and chemical composition of rosemary cutting. Journal of Plant Production, 8(8):797-803.

Miguel, M.G.; Guerrero, C.; Rodrigues, H. and Brito, J. (2007). Essential oils of Rosmarinus officinalis L., effect of harvesting dates, growing media and fertilizers. Proc. of The International Conference on Energy, Environment and Sustainable Development, Agios Nikolaos, Greece.

Moss, M.; Cook, J.; Wesnes, K. and Duckett, P. (2003). Aromas Rosemary and lavender essential oils differentially affect. Cognition and mood in healthy adults., Int. J. Neuro. Sci., 113:15-38.

Paradikovic, N.; Zeljkovic, S.; Kalec, M.T.; Vinkovic, T.; Dervić, I. and Marić, M. (2013). Influence of rooting powder on propagation of sage (Salvia officinalis L.) and rosemary (Rosemarinus officinalis) with green cuttings. Pojoprivreda, 19(2):10-15.

Paulus, D.; Valmorbida, R. and Paulus, E. (2016). Indole butyric acid on rosemary vegetative propagation. Horticulture Brasileira, 34:520-528.
Polumin, O. and Huxley, A. (1987). Flowers of the Mediterranean. Hogarth Press, London, UK, 272 p.

Raji, A.A. and Mohamad, O. (2012). Effect of stem cutting types, position and hormonal factors on rooting in Stevia rebaudiana bertoni. J. of Agricultural Science., 4(1):49-57.

Singh, M. and Guleria, N. (2013). Influence of harvesting stage and inorganic and organic fertilizers on yield and oil composition of rosemary (Rosmarinus officinalis L.) in a semi-arid tropical climate. Industrial Crops and Products, 42:37-40.

Snedecor, W.G. and Cochran, G.W. (1989). Statistical Methods, $8^{\text {th }}$ Ed., the Iowa State Univ. Press, Ames, Iowa, USA., $524 \mathrm{p}$.

Varban, D.I.; Varban, R.; Ghete, A.; Odgiu, A. and Czako, R.S. (2018). The influence of some products on the rooting of Rosmarinus officinalis L. cuttings. Hop and Medicinal Plants, 1-2:94-100.

Vasca-Zamfir, D.; Balan, D.; Luta, G., and Gherghina, E. (2018). Effect of rhizogenic biostemulators on Rosmarinus officinalis rooted cuttings biochemical composition. Scientific Papers Series B, Horticultur, 62:565-570.

\footnotetext{
تأثير منشط التجذير (فوس روت) مع استخدام أو بدون استخدام إندول حمض البيوتيريك على تجذير

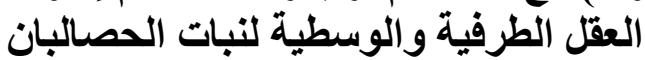




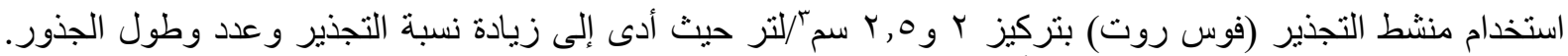

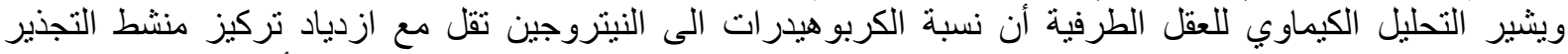

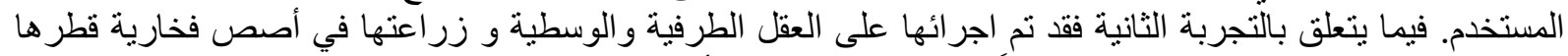

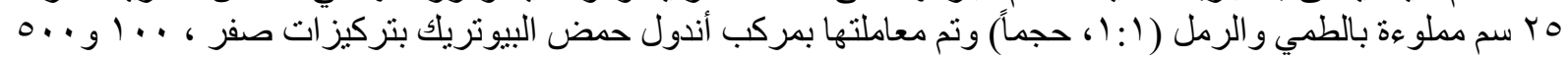

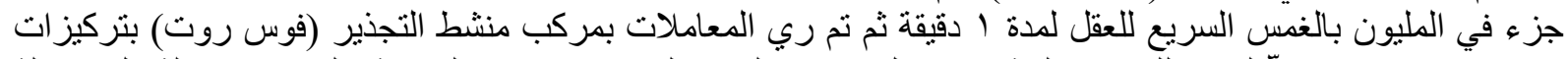

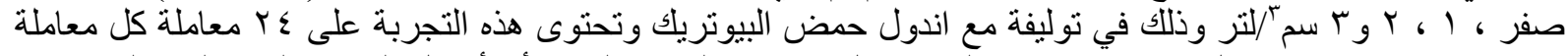

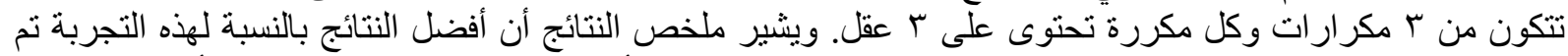

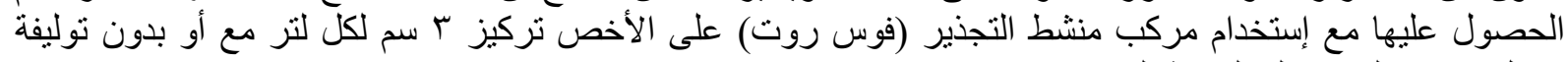
اندول حمض البيوتيريك السابقة الذكر. 